\title{
A cellular base station antenna configuration for variable coverage
}

\author{
Abdul-Rahman Shakeeb, K. H. Sayidmarie \\ Department of Communication Engineering, Ninevah University, Iraq
}

\begin{tabular}{l}
\hline Article Info \\
\hline Article history: \\
Received Jun 7, 2018 \\
Revised Oct 12, 2018 \\
Accepted Nov 11, 2018 \\
\hline Keywords: \\
Amplitude-only scanning \\
Base station coverage \\
Mobile systems \\
Reconfigurable antennas \\
Scanned arrays \\
Switched beam antennas \\
\hline
\end{tabular}

\begin{abstract}
The field coverage offered by the base station antenna in GSM systems influences the reception and interference performances. The coverage can be varied by scanning the mainbeam direction or varying the shape of the radiation pattern. In cellular system applications, a simple technique is desirable to achieve this goal. A simple technique to vary the coverage of cellular base station is investigated. The technique uses two conventional antennas tilted by a certain angle and fed by the same signal but at variable amplitudes. It is demonstrated that the field across one half of the covered sector can be gradually increased while that at the other half is reduced by varying the excitations of the two antenna elements. This can be deployed in a simple electronic means in response to the changing scenario rather readjusting the direction of the base station antenna.
\end{abstract}

Copyright $\odot 2019$ Institute of Advanced Engineering and Science. All rights reserved.

\section{Corresponding Author:}

Khalil Hassan Sayidmarie,

Department of Communication Engineering,

Ninevah University,

Mosul, Iraq.

Email: kh.sayidmarie@uoninevah.edu.iq

\section{INTRODUCTION}

Beam-steering antennas are used in many applications to increase the strength of the desired signal and decrease the effect of the undesired interfering signal leading to enhancement in the signal-to-interference ratio (SIR) [1]. The base station antenna is one of the applications which needs a smart technique to achieve beam-steering to improve the performance of the WLAN and cellular communication. Such improvements can be achieved by scanning or modifying the radiation pattern of the antenna. Various concepts for achieving beam steering have been investigated such as phase-only scanning, frequency scanning [2], [3]. These methods are dependent on supplying linear phase across the array elements. Phase shifters have some unwanted properties such as being bulky, having limited sweep range, and the effect of the operation frequency on the performance. The digital delay lines can offer better performance; however, they are fabricated to give few discrete phase values thus resulting in discrete scanning angles. Various configurations of beam forming networks have been presented for the improvement of base station coverage or enhancing the performance of the mobile communication systems [4], [5]. Optimization algorithms such as the particle swarm technique were used for beam-forming in array antennas used by mobile base stations [6]. Such technique is useful at the design stage, but it would be difficult to vary the radiation pattern according to the working scenario.

The frequency scanning may not be suitable in cellular systems where strict frequencies have to be used to avoid interferences. Another technique employed the variation of the amplitude excitations of the array elements, and it does not need frequency scanning, but it requires a fixed phase shifter [7]-[9]. In [7] the idea of amplitude scanning technique was investigated using a 3-element array, while in [8] a developed version of the technique was investigated. 
In [9] we presented two similar antennas which are tilted by a certain angle from a common base-line and fed through two lines. By controlling the excitations of the two antennas, the combined main beam can be steered from the normal to one antenna to that of the other antenna.

Scattering surfaces were employed to improve the radiation patterns of the base-station antenna so that the efficiency and coverage quality is enhanced. The obtained results were a reduction in the back lobe level and a slight improvement in the beam shape [10].

A dual beam array (DBA) for sectorizing the cellular site was presented in [11]. The DBA comprises a multi-column array and a beam forming circuit, which produces two overlapping beams with adjustable beam patterns. The arrangement furnishes the formation of two overlapping beams where the amplitude and phase excitations are implemented through the feed network. The radiation characteristics of the proposed antenna were investigated by simulation and experimentally. However, no information about the coverage of the antenna was given [11]. A method for capacity and coverage optimization using base station antenna electrical tilt in mobile networks has been recently proposed [12]. Development of a learning algorithm for automated antenna down tilting was utilized. The relationship between the base station antenna downtilt and the downlink network capacity in terms of the coverage probability and the area spectral efficiency have been investigated recently [13]. The vertical angle and horizontal angle of base station antenna based on two-dimensional electrically adjustable technology was presented to remote control the coverage area of the entire base station network. However, the antenna tilt and rotation are implemented by motors driven through software routines [14].

In[15] Jain, Mayank, and R. P. Agarwal showed the benefits of using multiple beams and differently shaped beams to improve the coverage \& capacity in a cell area with the maintenance of subscriber management within a cell sector for wireless communication. An alternative method to vary the antenna pattern of the base station antenna can be by placing a null in the radiation pattern. The null has to be directed towards the interfering source or a reflecting region. The introduced null in the pattern can be steered towards the desired direction by varying the excitation of the two side elements in the antenna array [16]. Such method was shown to provide a wide null with low sensitivity to frequency variation [17]. This technique is interesting as it only requires varying the excitations of two elements instead of changing the excitations of all the array elements.

In this contribution, the coverage on the land level achieved by the proposed antennas in [9] is investigated and how the coverage map can be modified utilizing the proposed amplitude beam scanning method. The proposed technique offers low complexity and cost for beam steering since the variation of magnitude is easier than the variation of phase or frequency. Moreover, it utilizes the conventional array antennas that are already in use in the base stations.

\section{THE PRINCIPLE OF AMPLITUDE SCANNING}

The idea of amplitude scanning [9] is illustrated in Figure 1 where two identical antennas are placed in a single plane and each is tilted by an angle $\psi$ from the common base line (X-axis). The idea of amplitude scanning is to supply variable amplitudes to the to the two antennas shown in Figure 1. One scheme is to increase the excitation of antennal by a certain amount while that given to antenna 2 is reduced by the same amount. Thus the amplitude excitations of the two antennas can be arranged as:

$$
\begin{aligned}
& \mathrm{a}_{1}=1+\Gamma \\
& \mathrm{a}_{2}=1-\Gamma
\end{aligned}
$$

The radiation pattern function of the two antennas can be given by

$$
\operatorname{AF}(\phi)=a_{1} F(\phi-\psi)+a_{2} F(\phi+\psi)
$$

Where $\mathrm{F}(\phi)$ is the amplitude pattern function of the two similar antennas.

The above assumption can be realized by using a power or amplitude dividers for transmitting array, or variable attenuators for receiving arrays. When the amplitude excitation of antenna1 is increased, and that of antenna2 is reduced by the same amount, the main beam of the radiation pattern will be directed towards the normal to antennal since it is supplied by larger excitation. Thus, the main beam can be scanned as the factor $\Gamma$ is varied.

In a practical implementation into mobile communication systems, the base station antenna will be a pair of antennas (antenna1 and antenna2) that are fed by signals proportional to $a_{1}$ and $a_{2}$. The arrangement can be in the geometry shown in Figure 2. The baseline of the two antennas is placed parallel to the ground 
surface, where beam scanning is requested to be in the horizontal plane. Each of the two antennas will be the conventional linear array that is placed in the vertical direction. The tilt angle can be fixed at the installation stage while $a_{1}$ and $a_{2}$ are varied during the operation to achieve scanning in the horizontal direction.

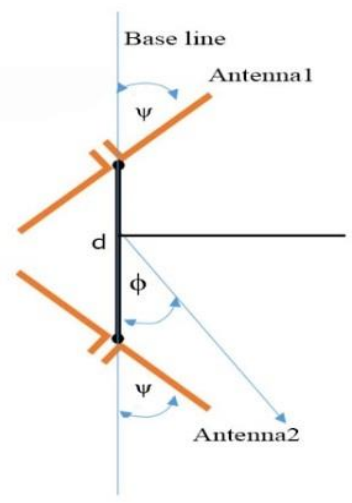

Figure 1. Geometry of the array formed of two antennas rotated by an angle $\psi$

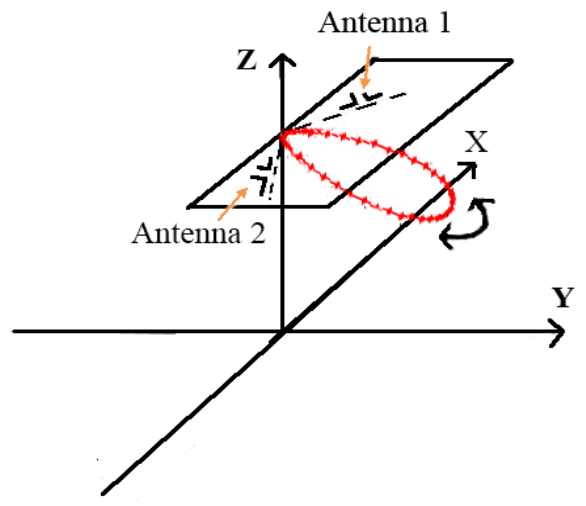

Figure 2. The geometry for beam scanning in the XY-plane

\section{SIMULATION OF THE COVERAGE}

In order to study the distribution of the signal level across the coverage area due to the proposed antennas, the covered area on a representative land was represented by a grid of 3321 points in the (x-y) plane as shown in Figure 3. A spacing between adjacent points of $50 \mathrm{~m}$ and an antenna height of $40 \mathrm{~m}$ were assumed. Concerning Figure 3, the range of $x$ was considered to be from - $2000 \mathrm{~m}$ to $2000 \mathrm{~m}$, while that for $\mathrm{y}$ was considered to be from 0 to $2000 \mathrm{~m}$. Thus, the antenna is assumed to have radiation across half plane which is a typical case for base stations scenarios.

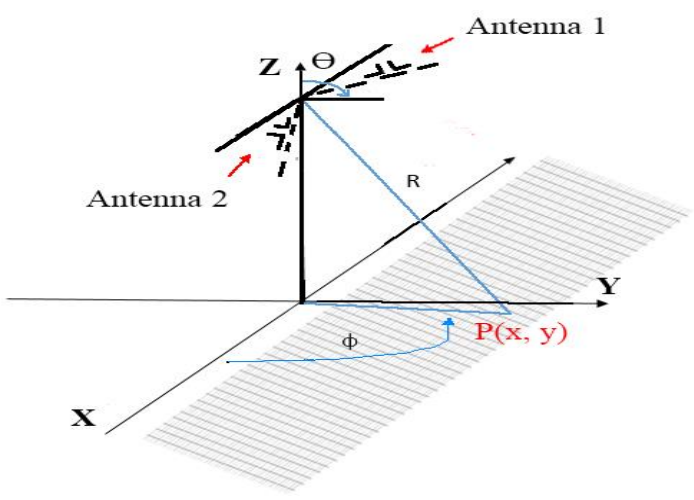

Figure 3. The geometry for a base station antenna and coverage area

As shown in Figure 3, the distance between the antenna and a typical point $\mathrm{p}(\mathrm{x}, \mathrm{y})$ on the covered ground is.

$$
\mathrm{R}=\sqrt{x^{2}+y^{2}+h^{2}}
$$

The free space propagation loss in the radiated signal varies inversely with the distance $\mathrm{R}$. This variation will be accounted for through the ratio $h / R$, where $h$ is the height of the base station antenna over the ground plane. The test point $\mathrm{p}(\mathrm{x}, \mathrm{y})$ can also be defined by the angles; $\theta$ is the down look from the normal to the antenna plane, and $\phi$ is the azimuth angle. The two angles can be given by: 


$$
\begin{aligned}
& \Theta=\tan ^{-1} \frac{\sqrt{x^{2}+y^{2}}}{h} \\
& \phi=\tan ^{-1}(y / x)
\end{aligned}
$$

To investigate the effect of scanning the radiation pattern on the coverage, the reflected signals from the ground and nearby objects were not considered in the following simulations. However, these reflections can be included in the simulations if the objects and buildings in the covered area are specified for a given site.

\section{RESULTS OF COMPUTER SIMULATIONS}

\subsection{The antenna with two half-wave dipoles}

The proposed idea was investigated by using two half-wave dipoles. This choice is in accordance with the conventional antennas used in mobile base stations where arrays of half wavelength dipoles are employed. Each half-wave dipole is oriented parallel to the ground plane as shown in 2 . The first case in the computer simulations assumed equal excitations for the two dipoles and the tilt angle $\psi$ was varied from zero to $60^{\circ}$ to explore the effect of this angle on the resulting radiation pattern. The obtained results for various values of the tilt angle are normalized to the maximum for the case $\psi=0^{\circ}$. Figure 4 shows a sample of the obtained coverage when the antenna elements are equally excited and the tilt angle $\psi=30^{\circ}$. The coverage is symmetrical with respect to the normal to the antenna base line, or in other words, it is symmetrical across the covered sector. It was found that as the tilt angle $(\psi)$ is increased from $0^{\circ}$ to $60^{\circ}$ the coverage becomes wider and both sides acquire a high level of received signal. It can be seen that across most of the shown area the signal level is between -25 to $-50 \mathrm{~dB}$ (these are relative to the maximum of $0 \mathrm{~dB}$ at $\psi=0^{\circ}$ ). The area in front and near to the base station antenna has the highest signal levels.

It should be noted that for equal excitations and zero tilt angle $(\psi)$ the two antennas form a conventional 2-element array, whose HPBW is smaller than that of a single antenna. Introducing the tilt angle $(\psi)$ results in a broader coverage. The choice of tilt angle $(\psi)$ can help in the design stage to obtain a suitable width of coverage. However, a single antenna with larger HPBW can be employed when scanning is not required.

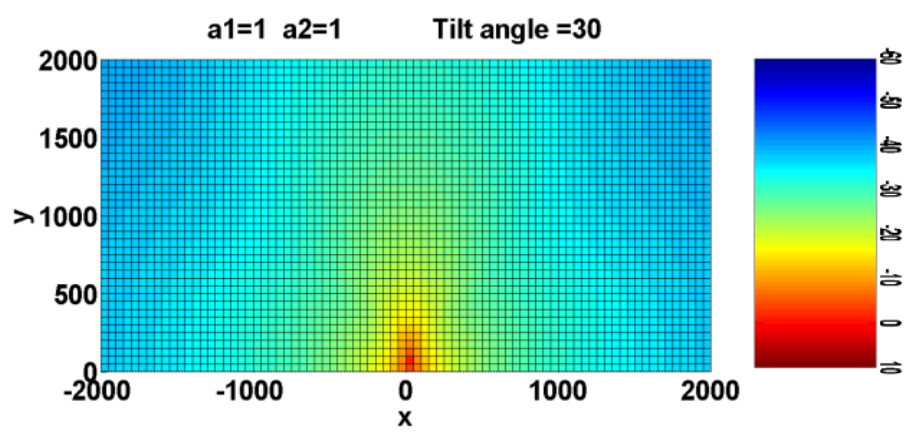

Figure 4. Coverage in the $X Y$ plane at tilt angle $=30^{\circ}$ and equal excitations $\left(a_{1}=a_{2}\right)$.

Figure 5 demonstrates how the coverage can be scanned from the normal direction (symmetric coverage) to clockwise and, counterclockwise directions. Various values of for the excitations $\left(a_{1}\right.$ and $\left.a_{2}\right)$ were considered while the tilt angle was fixed at $\left(30^{\circ}\right)$. It can be seen from the results that the coverage can be shifted from the normal to the antenna (when $\mathrm{a}_{1}=\mathrm{a}_{2}$ ) to either of the left or right directions. For example, when $a_{1}>a_{2}$ then the coverage is shifted to the right half of the covered sector as shown in Figure 5().

For a given tilt angle, the field at the left part of the coverage area increases as compared to that of the right part as the amplitude excitation of the left dipole is reduced and the amplitude excitation of the right dipole is increased as shown in Figure 6. 


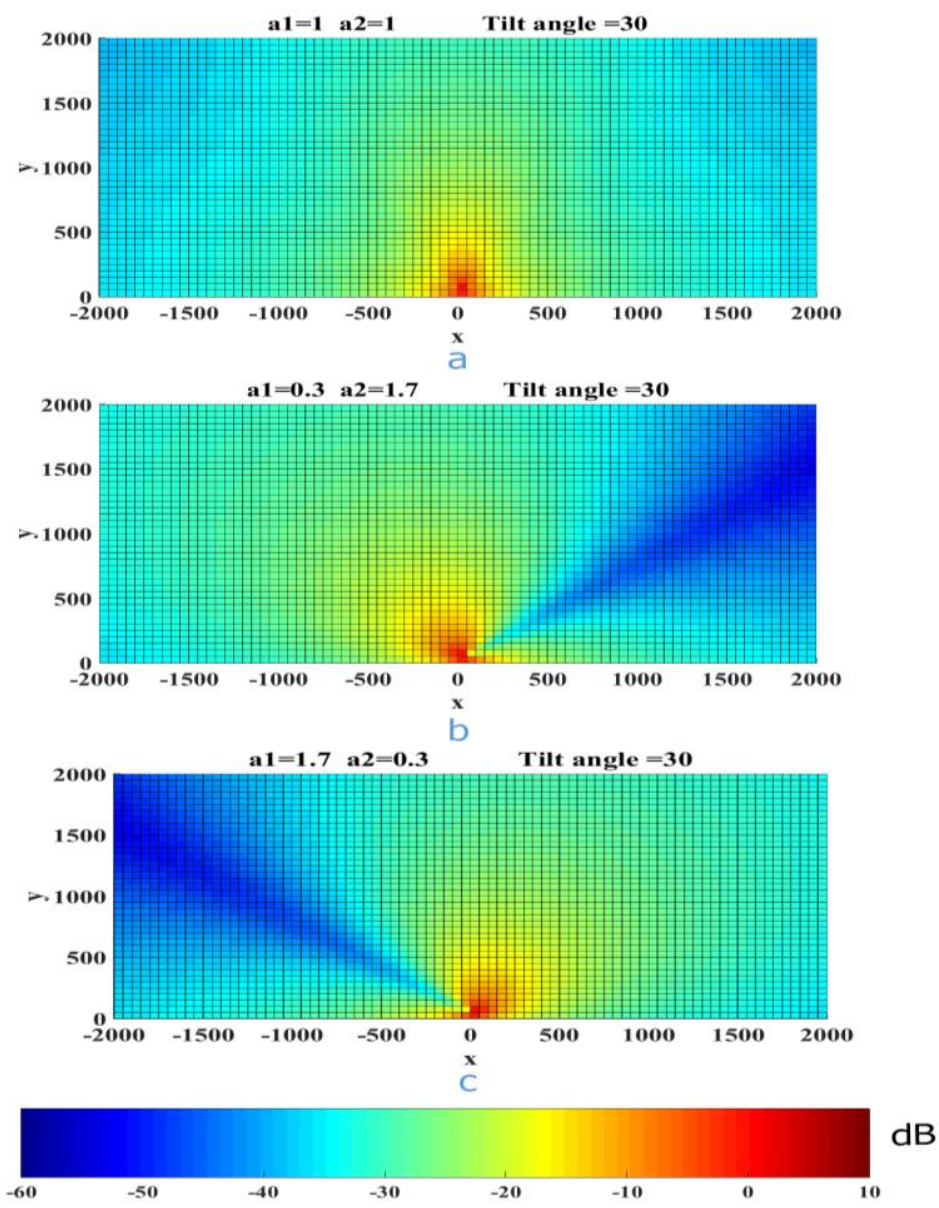

Figure 5. Coverage at the XY plane for various values of the amplitude excitation with tilt angle $(\Phi)=30^{\circ}$

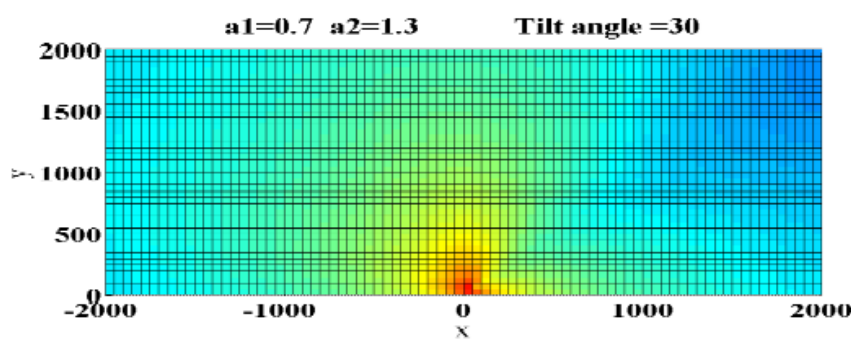

a

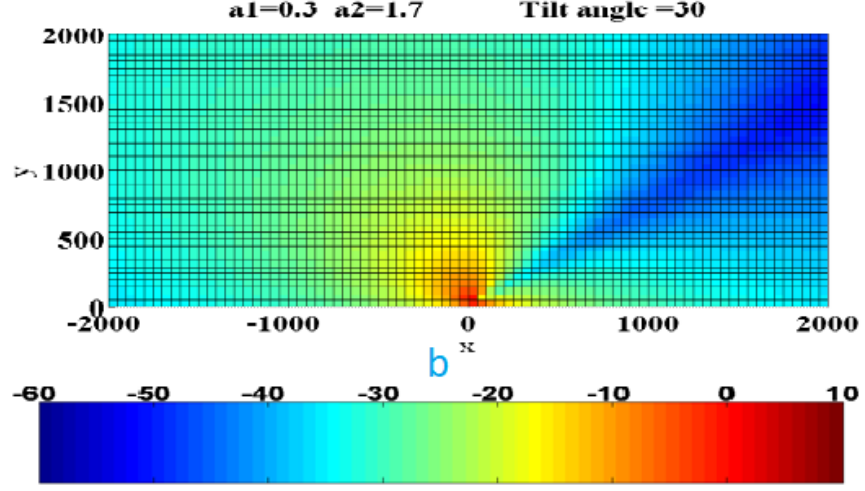

Figure 6. Coverage in the XY plane at tilt angle $=30^{\circ}$ and unequal excitations $\left(\mathrm{a}_{1} \neq \mathrm{a}_{2}\right)$ 
The results of Figure 4, Figure 5, and Figure 6 show that the tilt angle can be chosen at the design stage or the antenna deployment to obtain a pattern of desired beamwidth and coverage. The relative excitation can either be chosen at that stage or may be varied during the operation to vary the coverage to be more suitable to the changing scenario.

\subsection{The antenna with two microstrip elements}

The proposed antenna was implemented in further simulations where its two elements were assumed as microstrip antennas. Figure 7 shows the coverage area for the different values of the tilt angles $(\psi)$ for unequal amplitude excitation of the two microstrip antennas. The figure shows that the pattern is scanned towards the left as the tilt angle $(\psi)$ is increased. The signal levels on the left part are seen to increase while those at the right part decrease at tilt angle is increased.

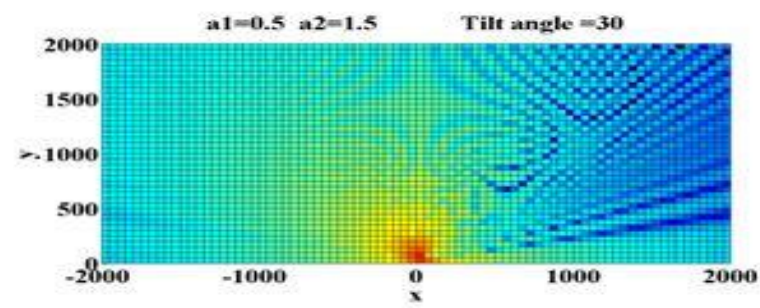

a

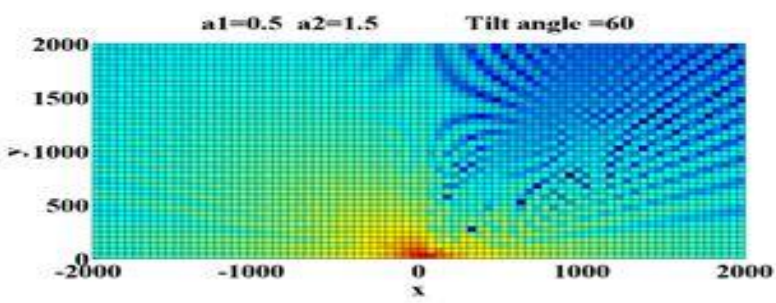

$\mathbf{b}$

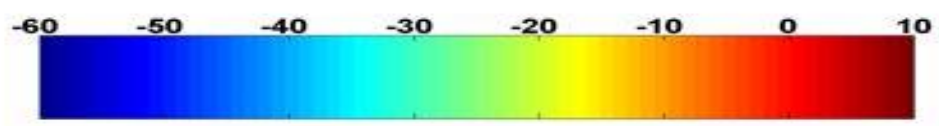

Figure 7. Coverage in the XY plane at tilt angle: $a=30^{\circ}, b=60^{\circ}$

\section{CONCLUSION}

The technique of amplitude scanning of the radiation pattern is implemented to vary the coverage of the base station antenna in the mobile communication system. The coverage of the proposed antenna was investigated, and the effect of two parameters (tilt angle and relative excitations) were explored. It has been demonstrated that the coverage of the base station antenna can be varied in a simple electronic way. The proposed technique offers low complexity and cost for beam steering since the variation of magnitude is easier than the variation of phase or frequency. It was demonstrated that the symmetrically distributed field across the coverage region could be easily modified to an unsymmetrical coverage. The field across one half of the covered sector can be gradually increased while that at the other half is reduced by varying the excitations of the two antenna elements. The change in the coverage can be achieved through beam scanning where the pattern and main beam almost keep their shape. Alternatively, pattern shaping can also be considered as a method to vary the antenna coverage. Also, significant improvements in the range and capacity are usually obtained by using beam steering technique in base station. 


\section{REFERENCES}

[1] Huang, Kao-Cheng, and D. J. Edwards," Millimetre wave antennas for gigabit wireless communications: a practical guide to design and analysis in a system context," John Wiley \& Sons, 2008.

[2] R. C. Hansen, "Phased Array Antennas," $2^{\text {nd }}$ Ed., John Wiley, 2009.

[3] R. J. Mailloux," Phased Array Antenna Handbook," $2^{\text {nd }}$ Ed., Artech House Inc., 2005.

[4] Deeksha Singh, M. Ganesh Madhan, A. Kamalaveni,"A compact passive beam forming network for base station antenna," IEEE International Conference on Microelectronic Devices, Circuits and Systems (ICMDCS), PP.10-12 Aug. 2017.

[5] Ardavan Rahimian," Microwave Beamforming Networks for Intelligent Transportation Systems," Chapter in the Book Intelligent Transportation Systems, PP. 123-142.

[6] Z. D. Zaharis,"Radiation pattern shaping of a mobile base station antenna array using a particle swarm optimization based technique," Electrical Engineering, vol. 90(4),PP. 301-311, 2008.

[7] Sayidmarie K. H., and Baedaa JM Jasem, "Amplitude-only beam scanning in linear antenna arrays," IEEE 7th International Multi-Conference on Systems Signals and Devices, SSD-2010, 2010.

[8] Sayidmarie K. H., and M.N. Saghurchy, "Array beam Scanning by Variation of Elements Amplitude-Only Excitations," IEEE 4th International Symposium on Microwave, Antenna, Propagation, and EMC Technologies for Wireless, MAPE 2011, PP. 749-753, nov 2011.

[9] Sayidmarie K.H., and Abdul-Rahman Shakeeb, "Array Beam Scanning and Switching by Variation of AmplitudeOnly Excitations", International Journal of Electromagnetics and Applications, vol.7(2), PP. 25-30, 2017.

[10] Cheng-Hsiung Wu, Chang-Fa Yang, Te-Shun Wang, Kuan-Chi Liao, Yen-Ming Chen, "A Method to Improve the Radiation Patterns of the Base-Station Sector Antenna for Mobile Communications by Adding Scattering Structures on the Front Panel of the Antenna," IEEE Antennas and Propagation Society International Symposium, Albuquerque, NM, USA, PP. 4357-4360, July 2006.

[11] Senglee Foo, Bill Vassilakis, "Adjustable Multi-Sector Cellular Base Station Antenna," International Journal on Advances in Networks and Services, vol. 2(1), PP. 25-41, 2009.

[12] Nikolay Dandanov, Hussein Al-Shatri, Anja Klein, Vladimir Poulkov, "Dynamic Self-Optimization of the Antenna Tilt for Best Trade-off Between Coverage and Capacity in Mobile Networks," Wireless Personal Communications, vol. 92(1), PP. 251-278, Jan 2017.

[13] Junnan Yang, Ming Ding, Guoqiang Mao, Zihuai Lin, De-gan Zhang, Tom Hao Luan, "Optimal Base Station Antenna Downtilt in Downlink Cellular Networks," Computing Research Repository, arXiv:1802.07479v1, Feb. 2018.

[14] Xi-Wang Dai, Zhen-Ye Wang, Cheng-Bin Zhang, and Hui Chen, "Remote control of base station antenna systems based on two-dimensional electrically adjustable technology," $7^{\text {th }}$ International ICST Conference on Communications and Networking in China (CHINACOM), PP.673-675, Aug. 2012.

[15] Jain, Mayank, and R. P. Agarwal. "Capacity \& coverage enhancement of wireless communication using smart antenna system," In Advances in Electrical, Electronics, Information, Communication and Bio-Informatics (AEEICB), 2016 2nd International Conference on, IEEE, pp. 310-313, 2016.

[16] Jafar R. Mohammed, and K. H. Sayidmarie, "A null steering method by controlling two elements," IET Microwaves, Antennas \& Propagation, vol. 8(15), PP.1348-1355, Dec. 2014.

[17] Sayidmarie K.H., and Jafar R. Mohammed, "Performance of a wide angle and wide band nulling method for phased arrays," Progress In Electromagnetics Research M, vol. 33, pp. 239-249, 2013.

\section{BIOGRAPHIES OF AUTHORS}

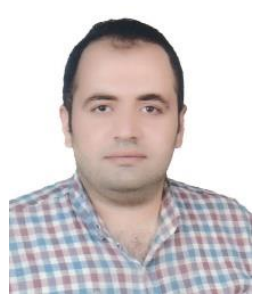

Abdul-Rahman Shakeeb received the B.Sc. degree Communication Engineering from the College of Electronic Engineering/Mosul University, Iraq, in 2012, and M.Sc. Degree in Communication Engineering from the College of Electronic Engineering/Mosul University, Iraq in 2017. His experience is in mobile communication systems.

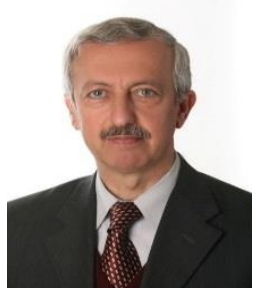

Prof Khalil Sayidmarie received the B.Sc. degree with first class honors in Electronic \& Communication Engineering from Mosul University, Iraq, in 1976, and Ph.D. Degree in Antennas \& Propagation from Sheffield University/ U.K. in 1981. Then he joined the College of Engineering at Mosul University in 1983, and was promoted to full professor on 1992. He worked as head of electrical engineering department for 9 years. He was a cofounder of the College of Electronic Engineering at Mosul University. He has been Professor of communication engineering at that college. Sayidmarie served as Prof. of communication engineering at the College of Engineering/ University of Amman Al-Ahliyya/ Jordan from Oct 2006 to Sept. 2009. He is professor of communication engineering at the College of Electronic Engineering/ Ninevah University. He has supervised $38 \mathrm{MSc}$ and $\mathrm{PhD}$ theses and published more than 120 papers. 